\title{
Agôn
}

Revue des arts de la scène

\section{5 | 2012}

L'entrée en scène

\section{L'entrée en scène du danseur : Rite(s) de passage(s)}

Entretiens réalisés par Claire Besuelle

Mario Thibodeau, Diane Leduc et Claire Bésuelle

\section{CpenEdition}

Journals

Édition électronique

URL : http://journals.openedition.org/agon/2426

DOI : 10.4000 /agon.2426

ISSN : 1961-8581

Éditeur

Association Agôn

Référence électronique

Mario Thibodeau, Diane Leduc et Claire Bésuelle, "L'entrée en scène du danseur : Rite(s) de

passage(s) », Agôn [En ligne], 5 | 2012, mis en ligne le 25 janvier 2013, consulté le 15 septembre 2020.

URL : http://journals.openedition.org/agon/2426

Ce document a été généré automatiquement le 15 septembre 2020.

Association Agôn et les auteurs des articles 


\title{
L'entrée en scène du danseur : Rite(s) de passage(s)
}

\author{
Entretiens réalisés par Claire Besuelle
}

Mario Thibodeau, Diane Leduc et Claire Bésuelle

\begin{abstract}
Danseurs-chercheurs, chercheurs-danseurs, Diane Leduc et Mario Thibodeau ont tous deux mis au centre de leur recherche universitaire le travail du danseur, avec la volonté de mieux en saisir les dynamiques internes. Mario Thibodeau revient ainsi sur son expérience de Premier Danseur au sein des ballets de Montréal dans une perspective psychologique, tandis que Diane Leduc tente elle une approche phénoménologique du travail de l'interprète en danse contemporaine. Deux perspectives, qui explorent les différents mécanismes inhérents au projet artistique du danseur, projet global dont l'entrée en scène constitue la charnière.

Quand le danseur entre en scène, qu'est-ce qu'il fait?

Concrètement : un pas, un saut, une glissade. Pourtant, bien plus profondément, une multitude de facteurs et de processus entrent en jeu dans ce franchissement du « seuil » entre la coulisse et la scène. Les études sont assez rares où l'accent est mis sur les processus, non seulement physiques ou esthétiques, mais aussi psychiques et émotionnels, qui entrent en jeu dans l'acte d'interprétation du danseur. C'est pourquoi il nous a paru particulièrement intéressant $d$ 'interroger ces deux chercheurs à propos du moment de l'entrée en scène, et de ce qu'il représente pour le danseur.
\end{abstract}

\section{« Apprivoiser le passage » - Mario Thibodeau}

Mario Thibodeau a été Premier Danseur pour le Ballet de Montréal pendant plus d'une quinzaine d'années, durant lesquelles il a connu une carrière internationale de grande envergure. Aujourd'hui, il poursuit une autre passion: la psychologie. Au titre de psychodynamicien analytique, il élabore en ce moment une thèse dont le thème principal traite du passage de l'artiste de la coulisse à la scène, qu'il envisage comme un véritable rite. Il a eu la gentillesse de patiemment partager avec nous l'état de ses recherches : une parole inédite et passionnée sur le travail de l'interprète. 


\section{Passer de la coulisse à la scène...}

Claire BESUELLE : Le passage de la coulisse à la scène est le cœur même de votre travail de thèse, qui consiste à tenter d'en expliciter les enjeux en termes de processus intrapsychiques...

Mario THIBODEAU: Le "passage " est un processus personnel, intime et propre à chaque artiste. Dans les dernières années de ma carrière, je me suis posé la question de la traversée de l'arrière-scène à la scène. Les spectateurs reçoivent une œuvre organisée, structurée et porteuse de sens. Pourtant, quelques minutes auparavant, juste avant l'entrée en scène de l'artiste, une forme de chaos s'installe! C'est le trac, les tensions nerveuses. En pensant au public, je me disais : "S'ils savaient ! ». C'est ce qui m'a poussé à m'interroger sur les modalités de ce passage chez l'artiste. J'ai questionné, plus précisément, ce que sont les processus inconscients, latents, que doit mobiliser l'artiste pour franchir le seuil, par l'entremise de l'approche psychodynamique analytique ${ }^{1}$.

$\mathrm{CB}$ : Donc, qu'est-ce qui est en jeu dans ce fameux «passage » et comment le qualifiezvous?

MT : Je me suis basé sur la schématisation séquentielle linéaire du rite de passage opérée par l'anthropologue Arnold Van Gennep ${ }^{2}$, qui consiste en l'identification de trois étapes du rite: préliminaire, liminaire, post-liminaire. Je les identifie à trois « lieux » distincts quand j'en viens à parler du passage du danseur à la scène. Ainsi, le stade préliminaire ou « monde profane ", c'est pour l'interprète " l'arrière-scène ", la loge, l'espace de préparation à la prise de rôle. Le stade liminaire, cet interstice entre deux mondes distincts et complémentaires, devient dans cette modélisation la coulisse, et enfin l'espace de la scène correspond au stade post-liminaire : à l'espace sacré. Par la suite, toujours dans cette perspective de modélisation du travail de l'interprète sur les processus rituels décrits par Van Gennep, j'ai associé à chaque étape $\mathrm{du}$ rite, du "passage » de l'arrière-scène vers la scène des processus intrapsychiques particuliers.

L'arrière-scène, d'abord: c'est le lieu de la métamorphose, et c'est sans doute la partie la plus difficile du passage. Hans-Georg Gadamer ${ }^{3}$ caractérise la métamorphose par la séparation : c'est « l'être antérieur » qui devient « nul et non avenu », dans un processus de disparition progressive du monde actuel, d'une dissolution de la temporalité. C'est le moment nécessaire, en fait, de la perte d'une part de la subjectivité de l'interprète : celle qui n'est pas nécessaire à l'interprétation du rôle.

\section{CB : Comment l'interprète se défait-il de sa subjectivité ?}

MT : Ce stade préliminaire de métamorphose est le lieu même du trac, d'une forme chaotique, des tensions nerveuses et psychiques. C'est connu : on éprouve un malaise, une forme de remise en question, de doute et des craintes qui peuvent aller jusqu'à certaines réactions psychosomatiques. Ces tensions nerveuses générées par le trac participent précisément à des déchirements et des fissures du psychisme qui paradoxalement aident et participent au processus de métamorphose. Il est important pour l'interprète alors d'accepter ce malaise et ces tensions internes qui vont faciliter la séparation entre la personne qui danse et l'artiste. Ce sont elles qui par la suite vont se transmuer en énergie pure, au service d'une création chorégraphique. 
Cette métamorphose, qui a lieu en amont, opère en même temps que deux « rites " qui s'intriquent l'un dans l'autre : un rite de purification et un rite funéraire. Un rite de purification opère pour se défaire des pensées négatives pour faire place au personnage. C'est opérer un processus de dépouillement et plus précisément de désidentification. Pour Van Gennep, on ôte ce qui est impur, ou on ajoute de la pureté, c'est selon. À la purification s'enchevêtre le rite funéraire qui équivaut à une forme de deuil : on fait précisément le deuil de certaines parties de soi, que l'on doit mettre de côté pour incarner le personnage.

\section{CB : Et ensuite ? Une fois que la métamorphose est « complétée » ?}

MT : On passe alors au stade liminaire : l'artiste se positionne en coulisse. Ce temps d'arrêt, au seuil de la scène, nécessite l'élaboration de deux autres processus qui sont presque simultanés : la transmutation et la translation.

La transmutation, c'est une transformation en totalité et qui s'opère très rapidement. Pour suivre Gadamer, il s'agit d'une transformation soudaine: "Ce qui existait auparavant n'existe plus, et ce qui existe maintenant est le vrai qui subsiste ». C'est à ce moment que l'interprète atteint la " plénitude de sens ${ }^{4}$ ». C'est capital : une œuvre d'art, quelle qu'elle soit, doit être porteuse de sens. L'artiste à ce moment-là connaît son rôle, ses pas, il maîtrise la technique. Il devient en totalité « contenu de sens » et révèle la réalité dans la vérité, l'essence originelle de la chorégraphie.

Mario Thibodeau, Cantate - Ballet de Montréal (1985)

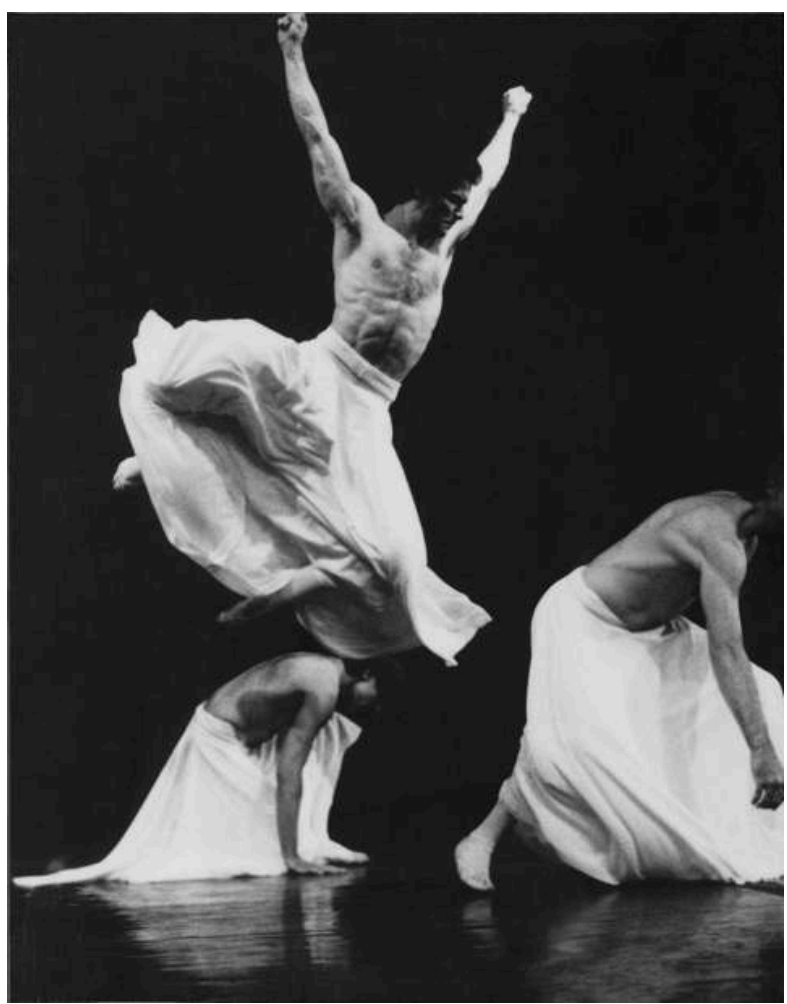

Collection privée

La translation, c'est un mouvement «bi-directionnel ». Je dirais que s'effectue, à la fois, un mouvement horizontal de la corporéité, de la coulisse vers la scène, et dans le même élan, un mouvement vertical de la pensée. C'est à partir de ce moment qu'un 
artiste peut ressentir parfois et vivre sur scène un état de sublimation pulsionnelle. Cette transmutation et cette translation ne sont rendues possibles que par un acte de foi, un engagement total de l'artiste. Je le vois comme un élan de la psyché, tournée en direction d'une autre vision du monde, un monde nouveau lié au sacré : l'œuvre d'art chorégraphique.

Le danseur passe alors au stade post-liminaire du rite, il est dans l'espace sacré de la scène. Van Gennep est clair : " passer le seuil signifie s'agréger à un monde nouveau ». L'interprète est alors transmué en "être de l'art ». Pour Gadamer ${ }^{5}$, le subjectum de l'œuvre prime sur la subjectivité de l'artiste. L'interprète dévoile la vision d'un monde nouveau aux spectateurs.

$\mathrm{CB}$ : Mais qu'est-ce qui reste alors de l'interprète ? De sa présence physique?

MT : C'est la présence du personnage, le Présenté comme le nomme Gadamer, qui se révèle aux spectateurs. Il faut y croire, sinon ça ne sert à rien. Dans un pas de deux avec Juliette, par exemple, je suis Roméo et j'aime réellement Juliette. Peu importe ce qui s'est passé avec ma partenaire en répétition, sur le plan physique ou relationnel. La seule chose qui compte lors de la présentation est l'œuvre.

CB: N'est-ce pas particulièrement difficile en danse, classique de surcroît, où la chorégraphie demande une telle précision, un tel investissement physique?

MT : Tout corps acquiert la morphologie de sa discipline. L'apprentissage du ballet forme ce que je nomme le corps-projection en fonction de la complexité et de l'exigence du travail corporel qui affine les lignes, et tend constamment au prolongement de ses membres dans l'espace. Toute forme de danse, tous styles confondus, exige l'apprentissage d'une technique, qui devient une seconde nature. Et tout danseur professionnel, je crois, prend conscience durant sa carrière, une fois la maîtrise de la technique acquise, que l'investissement psychique est plus exigeant et épuisant que les efforts physiques.

C'est pour cela que l'interprète se doit, avant de monter sur scène, de maîtriser sa discipline, à savoir : sa technique, son style et l'esthétisme de son art. Ça prend au moins généralement une dizaine d'années de travail assidu avant que l'artiste fasse ses premiers pas sur scène ! Il faut nécessairement que l'interprète ait "incorporé " la chorégraphie avant de monter sur scène.

\section{L'espace sacré de la scène}

\section{CB : Vous parlez d'espace sacré de la scène, c'est un mot fort !}

MT : J'ai oublié que c'était un mot fort! Pour moi c'est une évidence, je crois que c'en est une pour tout artiste. C'est le respect qui est dû à cet espace transitionnel, où on peut créer. Le sacré fait référence à un monde interdit accessible seulement aux initiés. Un lieu particulier où l'artiste des arts de la scène se soumet à un respect absolu. La scène est un lieu sacré. La salle et la scène sont deux endroits distincts et différents, deux espaces distincts. L'étude du passage, de l'entrée en scène, m'a amené à penser qu'il n'y a pas de communication entre l'artiste et le spectateur, mais une communion, qui s'opère par l'entremise du sens de l'œuvre, contenu par l'interprète, et reçu, capté par le spectateur. Maurice Béjart apporte à ce propos un éclairage intéressant, lorsqu'il mentionne que « l'art est un point de jonction entre le réel et le transcendant $»^{6}$ et le danseur en est le " médium ». Quelque chose émane de 
l'œuvre et nous parle, nous interpelle directement, et ce lien n'est pas de l'ordre de l'échange.

CB : En outre l'angle d'approche que vous adoptez pour parler du passage, de l'entrée en scène d'un artiste, est teinté de spiritualité...

MT : Effectivement, je ne parle pas de religiosité mais bien de spiritualité. C'est difficile de parler de cet acte de foi nécessaire à l'interprète et beaucoup de danseurs professionnels n'en diront rien, parce qu'on a peur - j'ai eu peur ! - de rendre ça public, de dévoiler mon «Dieu» sur la place publique et de révéler cette part d'intimité, de privé. On a peur qu'Il soit critiqué, jugé ou pire ridiculisé. Pourtant, il y a une part de spiritualité en moi, consciente ou inconsciente, dit Viktor Frankl ${ }^{7}$. C'est mon Dieu intime et personnel qui m'a toujours permis d'accomplir le passage.

\section{CB : On peut apprendre ça?}

MT : On peut l'apprivoiser. Encore une fois, c'est personnel et intime, propre à chaque artiste. J'ai réussi à dégager quelques grands axes, sur le plan des processus inconscients qui sous-tendent ce travail de l'artiste. Cela éclaire le phénomène, mais il reste profondément intime et propre à chaque artiste. Comme je le disais, avec les années, on peut apprivoiser le passage, ce trac-là. Mais il ne disparaît jamais. C'est la source, l'origine de l'énergie artistique, une énergie nécessaire à la création de l'œuvre et à sa présentation. Je parle de présentation parce que l'interprète est toujours dans l'essence de l'œuvre, jamais dans sa représentation. Je suis l'œuvre à chaque présentation, je ne la représente pas.

\section{$\mathrm{CB}$ : Et si on rate son passage?}

MT: On peut rater son passage. On peut rater son passage si on n'est pas suffisamment préparé... Entrer en scène sans être prêt, c'est se manquer de respect, à soi en tant qu'artiste-interprète, au public, et à l'espace de la scène, cet espace sacré. Si on ne réussit pas à réaliser les différentes étapes du passage, en considérant les processus intrapsychiques des transformations correspondant à chaque séquence du passage, le risque est que l'artiste traîne avec lui, dans son sillon, des traces du «trac », du chaos. Les tensions nerveuses générées par le trac n'auront pu alors se transformer, par la métamorphose et la transmutation, en énergie en vue d'une création artistique.

Il est important de comprendre que l'acte de foi est crucial pour réaliser le passage. Que l'œuvre chorégraphique soit narrative ou abstraite, il est impératif que l'œuvre touche le spectateur. L'un des pires scénarios serait la perte de sens de l'œuvre qui représente, de manière symbolique, la mort de l'artiste. Indépendamment de la raison, la foi, selon Françoise Dolto ${ }^{8}$, donne toujours un sens. C'est dans le silence et la noirceur de la coulisse, au seuil de la scène, que s'éveille le sens. La foi est l'élément déclencheur pour sortir du chaos et permettre d'entamer la naissance du mouvement créateur.

\section{Des « états modifiés de conscience » - Diane Leduc}

Diane Leduc s'intéresse à la danse depuis toute jeune. En tant qu'interprète, elle danse dans Maison, danses de chambre de Lucie Boissinot et dans Les Equinoxes, d'Alain Populaire. Elle collabore également avec Benoît Lachambre pour L'aberration des traces et Dédanses d'elles. En tant que chercheuse, elle a fait le choix de prolonger son 
parcours pratique : détentrice d'un doctorat en études et pratiques des arts, Diane Leduc s'intéresse tout particulièrement au travail et à la formation de l'interprète en danse contemporaine. Sa thèse porte sur l'étude phénoménologique de l'état d'authenticité dans l'acte d'interprétation en danse contemporaine. Riche de toutes ses expériences et des témoignages recueillis au cours de ses diverses recherches, elle a partagé avec nous ses réflexions autour du moment de l'entrée en scène, pour le danseur contemporain : en voici la synthèse.

\section{Préparer l'entrée : un point crucial dans la préparation de l'interprète}

Claire Besuelle: Selon vous et votre expérience, l'acte même d'entrer en scène peut-il s'apprendre ? Se préparer? Se travailler?

Diane LEDUC: Mon travail de thèse a démontré, me semble-t-il, que la préparation à la scène peut se faire de différentes manières ${ }^{9}$. Par des techniques d'abord : travail sur la concentration, l'intériorisation, la visualisation. Puis, par des répétitions rigoureuses, avec la reprise continuelle des éléments difficiles et des transitions. Enfin, par le développement de capacités à se mettre dans des états modifiés de conscience ${ }^{10}$. Ceux-ci sont à mon avis déterminants pour l'interprète puisqu'ils dicteront comment il se sentira sur scène : en confiance, tendu, détendu, énergique, etc. Ils équilibrent les tensions de toutes sortes et permettent de surmonter l'épreuve affective de la scène. Cet équilibre, toujours fragile, est une mise en relation du tendu et du souple, du confus et de l'harmonie, du contact à l'autre et de l'isolement, de la maitrise et de l'abandon. Ce sont eux les vrais outils de l'interprète. Travailler avec les états modifiés de conscience en répétition ne garantit toutefois pas qu'ils surviendront une fois sur scène. Ils aident à préparer les conditions de leur surgissement.

Donc, selon moi l'entrée en scène doit se préparer. Elle s'apprend et elle s'acquiert aussi par l'expérience. Certains interprètes laissent tomber des choses avec le temps. Ils acceptent parfois mieux avec l'âge de prendre plus de risques, de s'être trompés, de s'ouvrir aux imprévus. Ils apprennent aussi à vivre l'omniprésence

J'ajouterais que la formation des interprètes s'avère incomplète à ce sujet. La connaissance technique et physique est souvent le cœur du métier d'interprète au détriment des questions de concentration et les manières d'aborder la scène. Pourtant, au delà de l'œuvre, l'interprète est avant tout ce passeur particulier qui donne vie aux intentions de l'artiste concepteur. Tout un travail orienté directement sur la présence scénique permettrait à la danse contemporaine de profiter de l'expertise offerte dans des domaines connexes comme le théâtre.

CB: Le franchissement du seuil entre coulisse et scène et souvent vu comme la concrétisation d'une «prise de rôle » par l'interprète. En danse contemporaine, qu'en est-il de cet enjeu de l'incarnation?

DL : Pour moi, la concrétisation du rôle se fait bien en amont de l'entrée en scène. Elle survient au moment où la création est terminée et qu'il ne reste que du fignolage à faire. C'est cet instant où le chorégraphe dira : « la pièce est à vous maintenant ", où il s'en détache en quelque sorte. L'interprète n'a alors plus le choix : il doit prendre la pièce à bras le corps, l'assumer, l'ancrer dans son corps. Cependant, le rôle ne prend vie qu'une fois sur scène, qu'une fois exposé et vu par le public. 
Le rôle à interpréter est trop souvent basé sur le faire et le savoir-faire et trop peu sur le savoir-être. L'interprète est laissé seul avec la pièce dont il assume presque l'entière responsabilité, du moins quand il est sur scène. On ne lui apprend pas cela au cours de sa formation, il le découvre par lui-même, à tâtons. Ici encore, les états modifiés de conscience peuvent être d'un bon soutien parce que, pour les atteindre, l'interprète doit se questionner sur son rôle et il doit faire face à ses responsabilités. L'acte d'interprétation devrait toujours prendre le sens d'un devoir pour l'interprète. Une bonne performance est faite d'une série de choix et de circonstances. L'interprète a peu de pouvoir sur ces dernières, mais il en a beaucoup sur les décisions qu'il prend pour tendre vers une performance maximale.

\section{Le passage : l'interprète comme " métamorphoseur »}

CB : Vous avez travaillé sur la notion d'authenticité de l'interprète en danse contemporaine. II me semble, précisément, que le moment de l'entrée en scène cristallise de nombreux enjeux quant à la «présence » d'un interprète en scène, et qu'il la «modélise», en quelque sorte. En tant qu'interprète et chercheuse, quel est votre ressenti?

DL : Le terme " cristallisé » suggère quelque chose de bien ancré, voire de rigide. Or, l'entrée en scène est tout sauf rigide. L'interprète est dans une extrême fragilité avant de mettre le pied en avant et de s'exposer. Il lui faut s'ouvrir sur l'inconnu et plonger dans le noir avec la chorégraphie sous le bras. Cela dit, l'entrée en scène, ce bref moment où l'interprète fait le saut dans la lumière, est effectivement déterminante pour la qualité de la présence qu'il aura. Mais, aucune prédiction n'est possible ici. Parfois, ce sont les premières minutes d'échauffement mental qui feront en sorte que la performance sera ressentie comme réussie ou ratée. Parfois, c'est un incident qui donne spontanément une confiance non encore atteinte. Une mauvaise entrée peut provoquer par la suite un état modifié de conscience éblouissant.

Le moment d'entrée en scène n'est qu'un passage en somme. Si l'interprète est bien préparé, l'entrée en scène passera inaperçue pour lui (mais peut-être pas pour le public). Les interprètes que j'ai interrogées se préparent beaucoup avant la scène. En répétition évidemment, mais aussi au cours des heures qui précèdent le début du spectacle. Chaque rituel est différent, mais ils ont en commun une augmentation de la concentration. C'est d'ailleurs peut-être ce qui influe le plus sur la présence car outre le charisme et l'expérience, outre les prédispositions physiques et l'intelligence du corps, c'est la manière de voir la scène, de se la représenter qui compte. Comprendre pourquoi on fait ce métier, ce qui motive l'interprète à se placer dans ces moments de stress intense (la période de trac justement), génère une capacité à canaliser les énergies, à intérioriser les inquiétudes, à centraliser les aspects positifs.

CB : Que fait l'interprète, finalement, quand il entre en scène? Et plus personnellement peutêtre, pourquoi entre-t-on en scène, ou pour qui ?

DL : Les interprètes interrogées pour ma thèse vivent la scène comme un besoin essentiel dans leur vie. Elle entrent en scène pour elle-même, pour l'autre et pour la danse. Elles deviennent de véritables véhicules pour réconcilier des pôles et faire le pont entre la chorégraphie et le public.

Il me semble que le travail du danseur contemporain est avant tout de se sacrifier pour son art. Pas à n'importe quel prix bien sûr, par exemple pas au prix de sa santé physique. Mais, il y a tout de même une forme de sacrifice pour la danse et pour le 
public que l'interprète assume par le simple fait d'être interprète. Peut-être que le don de soi est plus juste parce qu'il fait ressortir davantage l'idée d'engagement (plutôt que l'idée de soumission, quoique ce dernier sujet est encore d'actualité en danse).

Quand on entre en scène, on se jette dans l'inconnu avec du connu. C'est comme s'il fallait transformer le connu en quelque chose de nouveau. En ce sens, l'interprète est réellement un passeur, un métamorphoseur. Ce moment de transformation est parfois synonyme de flottement. Durant quelques secondes ou quelques minutes, l'interprète passe d'un espace à un autre, de la coulisse à la scène, de l'isolement au public. Les perceptions fluctuent (le temps par exemple), l'espace changent, les sensations s'aiguisent. En fait, l'interprète danse aussi pour se transformer lui-même.

\section{« Être en scène »}

CB : Une fois le «seuil » franchi, comment appréhende-t-on la réalité d'être en scène, physiquement (d'un point de vue phénoménologique, par exemple)?

CL: Une fois sur scène, c'est la qualité du travail qui importe et les façons de le maintenir. La constance. À mon sens, l'investissement devra être total tout au long de l'exécution, quoi qu'il arrive. Pour y arriver, c'est tout un travail de consolidation qu'il faut faire bien avant la scène. L'expérience de scène peut aussi se décrire comme un parcours, une marche active à travers l'œuvre. Pour moi, une fois sur scène, après quelques gestes ou quelques minutes, le trac s'évacue pour laisser la place à la danse. L'interprète devrait à mon sens arriver à s'y abandonner. De toute façon, le corps sait ce qu'il faut faire. Il suffit de lui faire confiance et de se laisser prendre par lui. C'est en fait ce à quoi sert tout le travail de répétition: chaque geste, chaque sensation devient un automatisme disponible pour la danse. Plus besoin de réfléchir, simplement se laisser porter par le mouvement.

\section{$\mathrm{CB}$ : Comment gère-t-on le rapport au public, à un espace-temps différent?}

CL : Lorsque survient un état modifié de conscience, le rapport au public est simple : l'interprète est ouvert à ses réactions, les absorbe et en profite. Si l'état d'authenticité n'est pas présent, l'interprète est, selon moi, très vulnérable à l'énergie qui se dégage de la salle. Le public, quel qu'il soit, donne vie à l'œuvre dansée (danser dans son salon est sympathique, mais tant que la danse n'est pas vue, ce n'est pas de l'art). Nous avons surtout parlé du danseur, mais celui-ci est bien peu sans le public. C'est lui qui donne tout le sens à l'art et à la danse. Concernant l'espace-temps, il est différent selon les œuvres et les soirs de spectacle. Il n'est pas détaché $\mathrm{du}$ public. C'est un tout dans lequel l'interprète s'insère, comme un autre joueur.

\section{CB : Mario Thibodeau parle d'un «espace sacré » de la scène. Qu'en pensez-vous ?}

CL : Je vois la scène comme un espace ouvert sur le monde et un lieu rempli d'affectivité. Je ne le vois pas comme sacré en lui-même. Il est plutôt pour moi le lieu de l'accomplissement et de la communication. L'espace sacré est en soi, dans le corps de l'interprète, dans les traductions des messages qu'il porte. 


\section{NOTES}

1. Mario Thibodeau définit ainsi la psychodynamique analytique : Ce sont des applications de la théorie psychanalytique, constituée d'un ensemble de concepts organisés autour de la «métapsychologie» définie, essentiellement, comme l'ensemble des théories permettant de décrire un processus psychique sous trois rapports indissociables et inter-reliés: dynamique (conflits psychiques), économique (investissement psychique) et topique ( $1^{\mathrm{er}}$ : Conscient, Préconscient et Inconscient et $2^{\mathrm{e}}$ : Ça, Moi et Surmoi). Parmi les différentes approches en psychologie, l'approche psychodynamique est la seule que l'on nomme la "psychologie des profondeurs ». Elle est à la fois une théorie de la personnalité normale et pathologique, une méthode thérapeutique spécifique centrée sur l'interprétation des matériaux inconscients et une méthode de recherche qui permet de mettre en évidence le sens inconscient. Cette définition n'est pas succincte mais l'approche psychodynamique recouvre l'ensemble de processus intrapsychiques hypercomplexes qui ne peuvent être réduits à une psychologie (grec psukhê: âme et grec logos : parole, raison) basée sur une «science du comportement ». (référence : Brunet, Louis et Casoni, Diane. Profession psychologue. Montréal : Les Presses de l'Université de Montréal, 2009.)

2. Van Gennep, Arnold. Les Rites de passages. Paris : A\&J Picard, 2004. [1909]. Avec cet ouvrage, Van Gennep (1873-1957), que l'on peut considérer comme l'un des pères de l'ethnographie en France, met au jour un concept d'une importance décisive dans l'étude des sociétés humaines et de leur fonctionnement : la notion de rite de passage, « rites qui accompagnent les changements de lieu, d'état, d'occupation, de situation sociale, d'âge. Ils rythment le déroulement de la vie humaine, du berceau à la tombe ». (Belmont, Nicole. « Arnold Van Gennep » in Encyclopedia Universalis.

3. Gadamer, Hans-Georg. Vérité et méthode. Paris: Le Seuil, 1996. [1960]. P.129. Ouvrage fondamental de l'herméneutique contemporaine, paru en 1960 (réédité et augmenté en 1965 et 1972), Vérité et méthode, de Hans-Georg Gadamer (1900-2002) a profondément marqué le renouveau de la réflexion sur la notion de compréhension. Traité d'épistémologie qui tente de délivrer l'interprétation à l'œuvre dans les sciences humaines à la fois de l'objectivisme issu des sciences de la nature et du subjectivisme d'origine romantique, Vérité et méthode est aussi un traité de philosophie première. Si «l'être qui peut être compris est langue », c'est seulement à partir d'une connaissance des processus à l'œuvre dans le langage qu'une véritable anthropologie philosophique pourra être constituée. L'herméneutique ne peut se cantonner à une lecture des textes : elle doit s'ouvrir au monde lui-même. (Wybrands, Francis. "Vérité et méthode » in l'Encyclopedia Universalis.)

4. Gadamer, Hans-Georg. Op.cit. pp. 128-130.

5. Gadamer, Hans-Georg. Id., p.120

6. Béjart, Maurice. Lettres sur la danse de Noverre, présentées par Maurice Béjart. Paris : Ramsay, 1978. p. 23.

7. Frankl, Viktor Emil. Le Dieu inconscient. Psychothérapie et religion. Paris : éditions du Centurion, 1975. [1948]

8. Dolto, Françoise et Sévérin, Gérard. La Foi au risque de la psychanalyse. Paris : Le Seuil, 1981.

9. Leduc, Diane. Etude phénoménologique de l'état d'authenticité dans l'acte d'interprétation en danse contemporaine. Thèse de doctorat en études et pratiques des arts dirigée par Christine Hanrahan, Faculté des arts de l'Université du Québec à Montréal, département de danse, avril 2007.

10. Désigne tout état mental différent de l'état d'éveil ordinaire (notre mode de fonctionnement rationnel) : rêves, rêves lucides, états hypnotiques, états sophroniques, états hypnagogiques, états érotiques, rêverie, relaxation, interprétation artistique, intuition, créativité, transe, méditation, relaxation, transe, états mystiques... 
Ces états sont naturels (non pathologiques), même s'ils peuvent être provoqués par des techniques thérapeutiques spécifiques ou utilisés dans certaines méthodes du développement personnel. Ils correspondent à un ralentissement des ondes électriques du cerveau (des ondes bêta à l'état de veille, aux ondes delta pour le sommeil profond).

INDEX

Mots-clés : danse, corps, émotion 